Pacific Journal of Mathematic 


\title{
GENERALIZED CONVEXITY CONES AND THEIR DUALS
}

\section{DAN Amir AND Zvi ZiegleR}

\begin{abstract}
The structure of the intersections and unions of generalized convexity cones is analysed. The main results involve on the one hand denseness properties of sums of two distinct generalized convexity cones and on the other hand the availability of a decomposition for measures of the cones dual to intersections of generalized convexity cones. As an application an integration scheme which converges for all convex functions is found.
\end{abstract}

The generalized convexity cones, which were introduced by Karlin and Novikoff, find many applications in the systematic study of inequalities, in the theory of approximation and interpolation and in various branches of probability theory.

It is shown in $\S 1$ that the sums and differences of two generalized convexity cones are dense subsets of the Frechet space of continuous functions on the open interval $(a, b)$.

Passing to the dual cones in $\S 2$, we show that any measure belonging to the dual of the intersection of two (or, respectively, three) consecutive generalized convexity cones can be decomposed into the sum of two (respectively, three) measures belonging to the corresponding dual cones. For example, any measure belonging to the dual cone of the cone of convex and monotone functions can be decomposed into the sum of a measure belonging to the dual cone of the cone of convex functions and a measure belonging to the dual cone of the cone of monotone functions. It is also shown in this section that a decomposition of this type is not generally available for measures of the cone dual to the intersection of 4 consecutive generalized convexity cones.

In $\S 3$ we demonstrate the availability of a decomposition of a particularly simple type for measures of compact support on $(a, b)$ belonging to the cone dual to the intersection of two consecutive generalized convexity cones.

An application of the foregoing analysis, involving convergence properties of integration schemes, is given in $\S 4$.

Preliminaries. Let $\left\{u_{i}(t)\right\}_{i=0}^{N}$ be an Extended Complete Tchebycheff (ECT) system on the interval $[a, b]$ (for definitions of the pertinent concepts and for proofs of most of the statements in this section, the reader is referred to [3]). 
For $0 \leqq n \leqq N$, we denote by $C\left(u_{0}, \cdots, u_{n}\right)$ the convex cone of functions defined on $(a, b)$ which are convex with respect to $\left\{u_{i}\right\}_{0}^{n}$. The notation $C\left(u_{0}, \cdots, u_{-1}\right)$ is used to denote the convex cone $C^{+}$of nonnegative functions on $(a, b)$.

It is shown in [3, pp. 378-379] that in studying generalized convexity cones one may assume, with no loss of generality, that $\left\{u_{i}(t)\right\}_{i=0}^{N}$ are of the form:

$$
\left\{\begin{array}{l}
u_{0}(t)=w_{0}(t) \\
u_{1}(t)=w_{0}(t) \int_{a}^{t} w_{1}\left(x_{1}\right) d x_{1} \\
\vdots \\
\vdots \\
u_{N}(t)=w_{0}(t) \int_{a}^{t} w_{1}\left(x_{1}\right) \int_{a}^{x_{1}} w_{2}\left(x_{2}\right) \cdots \int_{a}^{x_{n-1}} w_{n}\left(x_{n}\right) d x_{n} \cdots d x_{1}
\end{array}\right.
$$

where the functions $w_{i}(t), i=0,1, \cdots, N$, are strictly positive on $[a, b]$ and $w_{i}(t) \in C^{N-i}[a, b], i=0,1, \cdots, N$. The first order differential operators

$$
D_{i} f(t)=\frac{d}{d t} \frac{1}{w_{i}(t)} f(t), \quad i=0,1, \cdots, N
$$

are closely related to the cones; in fact, if a function $f(t)$ is $n+1$ times differentiable, then it belongs to $C\left(u_{0}, \cdots, u_{n}\right)$ if, and only if $D_{n} D_{n-1} \cdots D_{0} f(t) \geqq 0, a<t<b$.

The cone $C\left(u_{0}, \cdots, u_{n}\right), 0 \leqq n \leqq N$, taken modulo the linear space spanned by $u_{0}, \cdots, u_{n}$, is spanned by a one-parameter family of extreme rays. These are represented by the functions $\phi_{n}(t ; x), a \leqq x \leqq b$, where $\phi_{n}(t ; x)$ is identically 0 for $a \leqq t<x$ and has the form

$$
u_{n}(t)+\sum_{i=0}^{n-1} c_{i}(x) u_{i}(t) \text { for } x \leqq t \leqq b
$$

(see [5], p. 566). The conjugate function $\tilde{\phi}_{n}(t ; x)$ is defined as

$$
(-1)^{n}\left[u_{n}(t)+\sum_{i=0}^{n-1} c_{i}(x) u_{i}(t)\right] \text { for } a \leqq t<x
$$

and 0 for $x \leqq t \leqq b$. By using the integral representation of $\tilde{\phi}_{n}(t ; x)$ (see [3], p. 387) and applying to it the operator $D_{n-1} D_{n-2} \cdots D_{0}$, it can easily be verified that $(-1)^{n} \widetilde{\phi}_{n}(t ; x) \in C\left(u_{0}, \cdots, u_{n-1}\right)$.

Let $M$ denote the space of Radon measures on $[a, b]$, i.e., the dual space of the Banach space $C[a, b]$ of continuous functions on $[a, b]$ with the sup norm. For a set $K$ of functions defined on $(a, b)$, let $K^{*}=\{\mu: \mu \varepsilon M, \mu(f) \geqq 0 \forall f \in K\} \quad\left(\mu(f)=\infty\right.$ is permitted). $K^{*}$ is clearly a convex cone, called the dual cone of $K$. We shall denote $\left[C\left(u_{0}, \cdots, u_{n}\right)\right]^{*}$ by $C^{*}\left(u_{0}, \cdots, u_{n}\right)$. The study of the extreme rays of 
convexity cones and of their intersections leads to the following characterization of dual cones ([5], p. 572):

Necessary and sufficient conditions for $\mu$ to belong to

$$
\left[\bigcap_{k=m}^{n} C\left(u_{0}, \cdots, u_{k}\right)\right]^{*}
$$

$(-1 \leqq m \leqq n \leqq N)$ are:

$$
\begin{cases}\int_{a}^{b} u_{i} d \mu=0 & i=0, \cdots, m, \\ \int_{a}^{b} u_{i} d \mu \geqq 0 \quad & i=m+1, \cdots, n, \\ \int_{a}^{b} \phi_{n}(t ; x) d \mu(t) \geqq 0, & a \leqq x \leqq b .\end{cases}
$$

Another useful characterization can be obtained along the lines described in ([2] and [6]). Define, for a measure $\mu \in M$, the functions

$$
\left\{\begin{array}{l}
\left(P_{0} \mu\right)(t)=\int_{t}^{b} w_{0}(x) d \mu(x), \\
\left(P_{j} \mu\right)(t)=\int_{t}^{b} w_{j}(x) P_{j-1} \mu(x) d x, \quad j=1,2, \cdots, N .
\end{array}\right.
$$

We then have:

Necessary and sufficient conditions for $\mu$ to belong to

$$
\left[\bigcap_{k=m}^{n} C\left(u_{0}, \cdots, u_{k}\right)\right]^{*}
$$

are:

$$
\begin{cases}P_{j} \mu(a)=0, & j=0,1, \cdots, m, \\ P_{j} \mu(a) \geqq 0, & j=m+1, \cdots, n, \\ P_{n} \mu(x) \geqq 0, & a \leqq x \leqq b .\end{cases}
$$

We shall avail ourselves also of the differential operators $D_{i}^{*}$, $i=0,1, \cdots, N$ defined by

$$
\left\{\begin{array}{l}
D_{0}^{*} f(t)=\frac{1}{w_{0}(t)} \frac{d^{R} f(t)}{d t} \quad \text { (right hand derivative) } \\
D_{i}^{*} f(t)=\frac{1}{w_{i}(t)} \frac{d f(t)}{d t}, \quad i=1,2, \cdots, N .
\end{array}\right.
$$

Note that if $f(b)=f^{\prime}(b)=\cdots=f^{(j)}(b)=0$, then $(-1)^{j+1} P_{j} D_{0}^{*} \cdots D_{j}^{*} f \equiv f$.

We finally recall that a simple example of an ECT-system is furnished by the system $1, t, t^{2}, \cdots$ on $[0,1]$. This corresponds to 
$\left\{w_{0} \equiv 1, w_{i} \equiv i, i=1,2, \cdots\right\}$. Convexity with respect to $\left(1, t, t^{2}, \cdots, t^{n}\right)$ is ordinary $n+1$-st order convexity. The extreme rays in this case are given by:

$$
\dot{\phi}_{n}(t ; x)=\left\{\begin{array}{cl}
0, & 0 \leqq t<x \\
(t-x)^{n}, & x \leqq t \leqq 1 .
\end{array}\right.
$$

1. Sums of generalized convexity cones. Let $C=C[a, b]$ denote the Banach space of real-valued continuous functions on the closed interval $[a, b]$, with the norm $\|f\|=\max \{|f(x)| ; a \leqq x \leqq b\}$. Let further $C_{0}=C(a, b)$ denote the Frechet space of continuous functions on the open interval $(a, b)$ with the topology of uniform convergence on compact subsets of $(a, b)$. The corresponding dual spaces are, respectively, the space $M$ of Radon measures $\mu$ defined on $[a, b]$ (these are representable as functions of bounded variation on $[a, b])$ and its subspace $M_{0}$ of Radon measures on $(a, b)$ with compact support-the duality being $\mu(f)=\int f d \mu$ (cf. [1]).

Theorem 1. The sums $C\left(u_{0}, \cdots, u_{n}\right)+C\left(u_{0}, \cdots, u_{m}\right)$, for $m>n \geqq 1$, and the differences $C\left(u_{0}, \cdots, u_{n}\right)-C\left(u_{0}, \cdots, u_{m}\right)$, for $m, n \geqq 1$, are dense (proper) subsets of $C_{0}$.

Proof. We recall first that, for $n \geqq 1$, every function of $C\left(u_{0}, \cdots, u_{n}\right)$ is continuous in $(a, b)$ (cf. [3]). Let $C^{\circ}\left(u_{0}, \cdots, u_{n}\right)$ denote the polar set of $C\left(u_{0}, \cdots, u_{n}\right)$ in $M_{0}$ i.e.,

$$
C^{\circ}\left(u_{0}, \cdots, u_{n}\right)=\left\{\mu: \mu(f) \geqq-1 \text { for all } f \in C\left(u_{0}, \cdots, u_{n}\right)\right\} \text {. }
$$

Since $C\left(u_{0}, \cdots, u_{n}\right)$ is a cone, this set coincides with the set $\left\{\mu: \mu(f) \geqq 0\right.$ for all $\left.f \in C\left(u_{0}, \cdots, u_{n}\right)\right\}$. Thus, we have

$$
C^{\circ}\left(u_{0}, \cdots, u_{n}\right)=M_{0} \cap C^{*}\left(u_{0}, \cdots, u_{n}\right) .
$$

Furthermore, since $C\left(u_{0}, \cdots, u_{n}\right)$ and $C\left(u_{0}, \cdots, u_{m}\right)$ are convex cones, their sum is the convex hull of their union, so that

$$
\begin{aligned}
& {\left[C\left(u_{0}, \cdots, u_{n}\right)+C\left(u_{0}, \cdots, u_{m}\right)\right]^{\circ}} \\
& \quad=\left[C\left(u_{0}, \cdots, u_{n}\right) \cup C\left(u_{0}, \cdots, u_{m}\right)\right]^{\circ} \\
& \quad=C^{\circ}\left(u_{0}, \cdots, u_{n}\right) \cap C^{\circ}\left(u_{0}, \cdots, u_{m}\right) \\
& \quad=M_{0} \cap C^{*}\left(u_{0}, \cdots, u_{n}\right) \cap C^{*}\left(u_{0}, \cdots, u_{m}\right) .
\end{aligned}
$$

In [6], Theorem 3 , it was proved that, for $m \neq n$,

$$
C^{*}\left(u_{0}, \cdots, u_{n}\right) \cap C^{*}\left(u_{0}, \cdots, u_{m}\right)=\{0\} .
$$

Thus, (1.1) implies that $\left[C\left(u_{0}, \cdots, u_{n}\right)+C\left(u_{0}, \cdots, u_{m}\right)\right]^{\circ}=\{0\}$ and 
$\left[C\left(u_{0}, \cdots, u_{n}\right)+C\left(u_{0}, \cdots, C_{m}\right)\right]^{\circ \circ}=C_{0}$. As $C\left(u_{0}, \cdots, u_{n}\right)+C\left(u_{0}, \cdots, u_{m}\right)$ is convex, the left hand side of this last equation is the weak-closure of $C\left(u_{0}, \cdots, u_{n}\right)+C\left(u_{0}, \cdots, u_{m}\right)$, which is also the strong closure by the Hahn-Banach theorem (cf. [1], 2.2.7). This proves that $C\left(u_{0}, \cdots, u_{n}\right)+C\left(u_{0}, \cdots, u_{m}\right)$ is a dense subset of $C_{0}$. It is a proper subset; moreover, its complement is dense as well. This observation follows from the fact that the functions of the convexity cones have one sided limits (possibly $\infty$ ) at $b$, while the functions which do not enjoy this property are obviously dense in $C_{0}$.

The proof of Theorem 3 in [6] shows also that, for every $m$ and $n, C^{*}\left(u_{0}, \cdots, u_{n}\right) \cap\left[-C^{*}\left(u_{0}, \cdots, u_{m}\right)\right]=\{0\}$, so that the corresponding result for $C\left(u_{0}, \cdots, u_{n}\right)-C\left(u_{0}, \cdots, u_{m}\right)$ follows in an exactly analogous way.

REmarks. (a) As $C_{0}$ is metrizable, Theorem 1 implies that, for every $f \in C_{0}$ and $m>n \geqq 1$, there exist two sequences $\left(g_{k}\right) \subset C\left(u_{0} \cdots, u_{n}\right)$ and $\left(h_{k}\right) \subset C\left(u_{0}, \cdots, u_{m}\right)$, such that $\left(g_{k}+h_{k}\right)$ converges to the given $f$ uniformly on each compact subset of $(a, b)$.

(b) Similar results hold when $m$ or $n<1$.

(c) Analogous results hold when $C_{0}$ is replaced by $C$ and $C\left(u_{0}, \cdots, u_{n}\right)$ by $C \cap C\left(u_{0}, \cdots, u_{n}\right)$.

2. Sums of dual cones in $M$. We know that each dual cone $C^{*}\left(u_{0}, \cdots, u_{n}\right)$ is closed in the weak $\sigma(M, C)$-topology of $M$. In this section we show that the sums of 2 or 3 consecutive dual cones are $\sigma(M, C)$-closed, while the sum of 4 consecutive dual cones need not be $\sigma(M, C)$-closed.

THEOREM 2. For each $n, 0 \leqq n \leqq N$, we have

$$
C^{*}\left(u_{0}, \cdots, u_{n-1}\right)+C^{*}\left(u_{0}, \cdots, u_{n}\right)=\left[C\left(u_{0}, \cdots, u_{n-1}\right) \cap C\left(u_{0}, \cdots, u_{n}\right)\right]^{*} .
$$

Proof. Since the inclusion relation

$$
C^{*}\left(u_{0}, \cdots, u_{n-1}\right)+C^{*}\left(u_{0}, \cdots, u_{n}\right) \subset\left[C\left(u_{0}, \cdots, u_{n-1}\right) \cap C\left(u_{0}, \cdots, u_{n}\right)\right]^{*}
$$

is obviously true, we need prove only the converse inclusion.

We start by analyzing the case $n=0$. Let a measure $\mu$, $\mu \in\left[C^{+} \cap C\left(u_{0}\right)\right]^{*}$ be given. By $(0.3)$ it satisfies

$$
\int_{a}^{b} u_{0}(t) d \mu(t) \geqq 0 ; \quad \int_{a}^{b} \dot{\phi}_{0}(t ; x) d \mu(t) \geqq 0, \quad a<x<b .
$$

Define now the measure $\mu_{1}$ by

$$
\mu_{1}=\left[\int_{a}^{b} u_{0}(t) d \mu(t)\right] \delta_{a}
$$


where $\delta_{a}$ is the Dirac measure with unit mass at $a$. Then clearly $\mu_{1} \geqq 0$, i.e., $\mu_{1} \in\left(C^{+}\right)^{*}$. Define now $\mu_{2}=\mu-\mu_{1}$. Then we clearly have

$$
\int_{a}^{b} u_{0}(t) d \mu_{2}(t)=\int_{a}^{b} u_{0}(t) d \mu(t)-\int_{a}^{b} u_{0}(t) d \mu_{1}(t)=0
$$

and

$$
\int_{a}^{b} \phi_{0}(t ; x) d \mu_{2}(t)=\int_{a}^{b} \phi_{0}(t ; x) d \mu(t) \geqq 0, \quad a<x<b .
$$

Hence, $\mu_{2} \in C^{*}\left(u_{0}\right)$, and the decomposition has been accomplished.

Assume now that $n \geqq 1$. Let a measure $\mu$,

$$
\mu \in\left[C\left(u_{0}, \cdots, u_{n-1}\right) \cap C\left(u_{0}, \cdots, u_{n}\right)\right]^{*}
$$

be given. By (0.5) it satisfies

$$
P_{j} \mu(a)=0, \quad i=0,1, \cdots, n-1,
$$

and

$$
P_{n} \mu(x) \geqq 0, \quad a \leqq x \leqq b .
$$

If $P_{n} \mu(a)=0$, then we already have $\mu \in C^{*}\left(u_{0}, \cdots, u_{n}\right)$; thus we may assume that $P_{n} \mu(\alpha)=A>0$. Define the function $F(t)$ by

$$
F(t)=A-v(t)
$$

where

$$
v(t)=\int_{a}^{t} w_{n}\left(x_{n}\right) \int_{a}^{x_{n}} w_{n-1}\left(x_{n-1}\right) \cdots \int_{a}^{x_{2}} w_{1}\left(x_{1}\right) \cdot 2\left|P_{0} \mu\left(x_{1}\right)\right| d x_{1} \cdots d x_{n} .
$$

Let $s=\inf$ \{support $\mu$; then $v(t) \equiv 0$ for $a \leqq t \leqq s$, and $v(t)$ is strictly increasing for $s \leqq t \leqq b$. Thus, $F(t) \equiv A$ for $a \leqq t \leqq s$, and $F(t)$ is a strictly decreasing function for $s \leqq t \leqq b$. Making use of conditions (2.1), we find

$$
P_{j} \mu(t)=-\int_{a}^{t} w_{j}(x) P_{j-1} \mu(x) d x, \quad i=0,1, \cdots, n-1
$$

where $P_{-1} \mu \equiv \mu$. This relation implies that

$$
\begin{aligned}
P_{n} \mu(t) & -P_{n} \mu(a) \\
= & (-1)^{n} \int_{a}^{t} w_{n}\left(x_{n}\right) \int_{a}^{x_{n}} w_{n-1}\left(x_{n-1}\right) \cdots \int_{a}^{x_{2}} w_{1}\left(x_{1}\right) P_{0} \mu\left(x_{1}\right) d x_{1} \cdots d x_{n}
\end{aligned}
$$

so that 


$$
\begin{aligned}
& P_{n} \mu(t)-F(t) \\
& \quad=\int_{a}^{t} w_{n}\left(x_{n}\right) \cdots \int_{a}^{x_{2}} w_{1}\left(x_{1}\right)\left[2\left|P_{0} \mu\left(x_{1}\right)\right|+(-1)^{n} P_{0} \mu\left(x_{1}\right)\right] d x_{1} \cdots d x_{n}
\end{aligned}
$$

is a nondecreasing nonnegative function, which is strictly positive and strictly increasing for $t>s$. Since $P_{n} \mu(b)=0$, it follows that $F(b)<0$. Since $F(t)$ is a strictly decreasing function for $s \leqq t \leqq b$, we may consider the inverse function $F^{-1}(t)$ on $[s, b]$. Let $c, s<c<b$ be defined by $c=F^{-1}(0)$, and introduce the function $F_{+}(t)=\max \{F(t), 0\}$. This function coincides with $F(t)$ on $[a, c]$ and is identically zero on $[c, b]$. Thus, we have

$$
P_{n} \mu(t) \geqq F_{+}(t)
$$

with strict inequality holding for a neighbourhood of $c$. Thus, there exist an $r, c-s>r>0$, and an $\eta>0$ such that

$$
P_{n} \mu(t)-F_{+}(t) \geqq \eta>0 \text { for } c-r \leqq t \leqq c+r .
$$

We introduce the smoothing kernels $\rho_{\varepsilon}(t)$ defined by

$$
\rho_{\varepsilon}(t)=\left\{\begin{array}{cl}
\alpha_{s} \exp \left[-\frac{1}{1-(t / \varepsilon)^{2}}\right], & |t|<\varepsilon, \\
0 \quad & |t| \geqq \varepsilon,
\end{array}\right.
$$

where $\alpha_{\varepsilon}$ is a normalizing factor, chosen so that

$$
\int_{-\infty}^{\infty} \rho_{\varepsilon}(t) d t=1
$$

Define now the function

$$
f(v)=\left\{\begin{array}{cl}
A-v, & -\infty<v \leqq A \\
0 & A \leqq v<\infty
\end{array}\right.
$$

and set

$$
f_{1}(v)=\int_{-\infty}^{\infty} f(t) \rho_{\varepsilon}(v-t) d t
$$

where $\varepsilon$ is chosen so small that $0<\varepsilon<\eta, F(c+r)<-\varepsilon$ and $F(c-r)>\varepsilon$.

It is easily seen that $f_{1}(v)$ is a monotone decreasing function since $f(v)$ is such. Furthermore, since $f(v)$ is piecewise linear, we have

$$
f_{1}(v)=A-v \text { for } v \leqq A-\varepsilon ; \quad f_{1}(v)=0 \text { for } v \geqq A+\varepsilon .
$$

By the choice of $\varepsilon$ and the fact that the application of $\rho_{\varepsilon}(t)$ amounts to an averaging operation, we find that 


$$
\left|f(v)-f_{1}(v)\right| \leqq \eta, \text { for all } v
$$

Setting $F_{1}(t)=f_{1}(v(t))$, we obtain

$$
\left|F_{+}(t)-F_{1}(t)\right| \leqq \eta \text { for all } a \leqq t \leqq b .
$$

Furthermore, $F_{1}(t) \equiv F(t)$ for $\left[a, F^{-1}(\varepsilon)\right]$, and $F_{1} \equiv 0$ for $\left[F^{-1}(-\varepsilon), b\right]$. By virtue of the choice of $\varepsilon$, and the fact that $F(t)$ is monotone decreasing, we have

$$
F_{1}(t) \equiv F_{+}(t), \quad \text { for } \quad t \in[a, c-r] \cup[c+r, b] .
$$

Note further that $F_{1}(t)$ is monotone decreasing. Since $f_{1}(v)$ is infinitely differentiable as a function of $v$, we deduce that $F_{1}(t)$ has continuous derivatives up to the $n$-th order, and an $n+1$-st right derivative (measure).

Construct the measure $\mu_{1}$ by

$$
\mu_{1}=(-1)^{n+1} D_{0}^{*} \cdots D_{n}^{*} F_{1} .
$$

Using (0.7), we have

$$
P_{j} \mu_{1}=(-1)^{n-j} D_{j+1}^{*} \cdots D_{n}^{*} F_{1} \quad 0 \leqq j \leqq n
$$

where for $j=n$ the right hand side is understood as $F_{1}$. These relations in turn imply that

$$
P_{j} \mu_{1}(\alpha)=(-1)^{n-j} D_{j+1}^{*} \cdots D_{n}^{*} F(\alpha)=0, \quad j=0,1, \cdots n-1
$$

and that

$$
P_{n-1} \mu_{1}(x)=-\frac{1}{w_{n}(x)} \frac{d F_{1}}{d x}
$$

which is nonnegative for $a \leqq x \leqq b$, by virtue of the monotonicity of $F_{1}(x)$. Relations (2.7) and (2.8) imply, by $(0.5)$, that $\mu_{1} \in C^{*}\left(u_{0}, \cdots, u_{n-1}\right)$.

Consider now the measure $\mu_{2}=\mu-\mu_{1}$. By virtue of (2.7) we obtain, using the fact that $P_{n} \mu_{1}(a)=F_{1}(a)=A$, that

$$
P_{j} \mu_{2}(a)=0, \quad j=0,1, \cdots, n .
$$

Furthermore, by using (2.3), (2.5) and (2.6), we deduce the nonnegativity, for all $a \leqq x \leqq b$, of

$$
P_{n} \mu_{2}(x)=P_{n} \mu(x)-P_{n} \mu_{1}(x)=P_{n} \mu(x)-F_{1}(x) .
$$

This, taken together with (2.9) and (0.5), shows that $\mu_{2} \in C^{*}\left(u_{0}, \cdots, u_{n}\right)$ and the decomposition has been accomplished.

THEOREM 3. For every $n, 0 \leqq n \leqq N-1$, we have 


$$
\sum_{k=-1}^{1} C^{*}\left(u_{0}, \cdots, u_{n+k}\right)=\left[\bigcap_{k=-1}^{1} C\left(u_{0}, \cdots, u_{n+k}\right)\right]^{*} \cdot
$$

Proof. As in the proof of Theorem 2, we need prove only that

$$
\sum_{k=-1}^{1} C^{*}\left(u_{0}, \cdots, u_{n+k}\right) \supset\left[\bigcap_{k=-1}^{1} C\left(u_{0}, \cdots, u_{n+k}\right)\right]^{*}
$$

since the other inclusion is obvious. Furthermore, using Theorem 2 we deduce that it suffices to prove that

$$
\begin{aligned}
& \quad\left[\bigcap_{k=-1}^{1} C\left(u_{0}, \cdots, u_{n+k}\right)\right]^{*} \\
& \quad \subset C^{*}\left(u_{0}, \cdots, u_{n-1}\right)+\left[C\left(u_{0}, \cdots, u_{n}\right) \cap C\left(u_{0}, \cdots, u_{n+1}\right)\right]^{*} .
\end{aligned}
$$

We start with the case $n=0$. Let a measure

$$
\mu \in\left[C^{+} \cap C\left(u_{0}\right) \cap C\left(u_{0}, u_{1}\right)\right]^{*}
$$

be given. By $(0.3), \mu$ satisfies the conditions

$$
\int_{a}^{b} u_{i}(t) d \mu(t) \geqq 0, \quad i=0,1,
$$

and

$$
\int_{a}^{b} \dot{\phi}_{1}(t ; x) d \mu(t) \geqq 0 \quad a<x<b .
$$

Define the measure $\mu_{1}$ by

$$
\mu_{1}=\left[\int_{a}^{b} u_{0}(t) d \mu(t)\right] \delta_{a}
$$

where $\delta_{a}$ is the Dirac measure with unit mass at $a$. Then we clearly see that $\mu_{1} \in\left(C^{+}\right)^{*}$ while $\nu=\mu-\mu_{1}$ satisfies

$$
\int_{a}^{b} u_{0}(t) d \nu(t)=0
$$

and

$$
\int_{a}^{b} \phi_{1}(t ; x) d \nu(t)=\int_{a}^{b} \phi_{1}(t ; x) d \mu(t) \geqq 0, \quad a<x<b
$$

so that, by $(0.3), \nu \in\left[C\left(u_{0}\right) \cap C\left(u_{0}, u_{1}\right)\right]^{*}$ and the decomposition has been accomplished for this case.

Assume now that $n \geqq 1$ and let $\mu \in\left[\bigcap_{k=-1}^{1} C\left(u_{0}, \cdots, u_{n+k}\right)\right]^{*}$ be given. By (0.3), it satisfies

$$
\left\{\begin{array}{l}
P_{j} \mu(a)=0 \quad j=0,1, \cdots, n-1 \\
P_{n} \mu(a) \geqq 0, \\
P_{n+1} \mu(x) \geqq 0, \quad a \leqq x \leqq b .
\end{array}\right.
$$


If $P_{n} \mu(\alpha)=0$, then the conditions (2.11) imply, by $(0.3)$ that we already have $\mu \in\left[C\left(u_{0}, \cdots, u_{n}\right) \cap C\left(u_{0}, \cdots, u_{n+1}\right)\right]^{*}$. Thus, we may assume that $P_{n} \mu(\alpha)=A>0$. Furthermore, since $P_{n+1} \mu(x) \geqq 0$ and

$$
\frac{d}{d x} P_{n+1} \mu(a)=-w_{n}(a) P_{n} \mu(a)<0,
$$

we deduce that $P_{n+1} \mu(\alpha)=B>0$.

Since $P_{n+1} \mu(x)$ and $w_{n+1}(t)$ are continuous, there exist a $c$ and an $\eta>0$ such that $a<c-\eta<c+\eta<b$ and

$$
\left\{\begin{array}{l}
P_{n+1} \mu(x) \geqq \frac{1}{2} B \text { for } a \leqq x \leqq c+\eta \\
C=\int_{a}^{c} w_{n+1}(t) d t \leqq B / 4 A
\end{array}\right.
$$

Set

$$
v(x)=\int_{a}^{x} w_{n+1}(t) d t, \quad F(x)=\left\{\begin{array}{cl}
A(C-v(x)) & a \leqq x \leqq c \\
0 & c \leqq x \leqq b
\end{array} .\right.
$$

Then $F(x)$ has the following properties:

$$
\begin{gathered}
P_{n+1} \mu(x) \geqq F(x), \quad a \leqq x \leqq b, \\
P_{n+1} \mu(x)-F(x) \geqq \frac{1}{4} B, \quad a \leqq x \leqq c+\eta, \\
D_{i}^{*} \cdots D_{n+1}^{*} F(a)=0, \quad i=0,1, \cdots, n, \\
-D_{n+1}^{*} F(a)=A .
\end{gathered}
$$

Define the function $f(v)$ by

$$
f(v)=\left\{\begin{array}{cl}
A(C-v) & -\infty<v \leqq C \\
0 & C \leqq v<\infty
\end{array}\right.
$$

and let

$$
f(v)=\int_{-\infty}^{\infty} f(t) \rho_{\varepsilon}(v-t) d t
$$

where $\rho_{\varepsilon}(t)$ is the function defined in (2.4) and $\varepsilon$ is chosen so that it satisfies

$$
\varepsilon \leqq \int_{c}^{c+\eta} w_{n+1}(t) d t, \quad \varepsilon \leqq \int_{c-\eta}^{c} w_{n+1}(t) d t, \quad A \varepsilon<\frac{1}{4} B .
$$

The function $f_{1}(v)$ is an infinitely differentiable, monotone nonincreasing and convex function of $v$. Moreover, we clearly have: 


$$
\begin{cases}f_{1}(v)=A(C-v), & v \leqq C-\varepsilon, \\ f_{1}(v)=0 \quad & v \geqq C+\varepsilon, \\ \left|f_{1}-f\right| \leqq \frac{1}{4} B, & C-\varepsilon \leqq v \leqq C+\varepsilon .\end{cases}
$$

Construct now the function $F_{1}(x)=f_{1}(v(x))$. Then, by using (2.17) and (2.18), we find

$$
\begin{cases}F_{1}(x)=F(x) \quad, & a \leqq x \leqq c-\eta, \\ F_{1}(x)=F(x)=0 \quad, & c+\eta \leqq x \leqq b, \\ \left|F_{1}(x)-F(x)\right| \leqq \frac{1}{4} B, & a \leqq x \leqq b .\end{cases}
$$

Furthermore, since $f_{1}(v)$ is a convex function of $v$, we have

$$
D_{n}^{*} D_{n+1}^{*} F_{1}=D_{n}^{*}\left[\frac{d f_{1}}{d v}\right]=\frac{w_{n+1}}{w_{n}} \frac{d^{2} f_{1}}{d v^{2}} \geqq 0 .
$$

Construct now the measure $\mu_{1}$ by: $\mu_{1}=(-1)^{n+2} D_{0}^{*} \cdots D_{n+1}^{*} F_{1}$. We have then $P_{j} \mu_{1}=(-1)^{n-j+1} D_{j+1}^{*} \cdots D_{n+1}^{*} F_{1}, j=0,1, \cdots, n$, and $P_{n+1} \mu_{1}=F_{1}$. Thus, by (2.15) and (2.19)

$$
P_{j} \mu_{1}(a)=(-1)^{n-j+1} D_{j+1}^{*}, \cdots, D_{n+1}^{*} F_{1}(a)=0, j=0,1, \cdots, n-1
$$
and, by $(2.20)$

$$
P_{n-1} \mu_{1}(x)=D_{n}^{*} D_{n+1}^{*} F_{1}(x) \geqq 0 .
$$

These relations imply by $(0.5)$ that $\mu_{1} \in C^{*}\left(u_{0}, \cdots, u_{n-1}\right)$.

Define now $\nu$ by $\nu=\mu-\mu_{1}$. Then, using (2.16), (2.14) and (2.19) we have

$$
\left\{\begin{array}{l}
P_{j} \nu(a)=P_{j} \mu(a)-P_{j} \mu_{1}(a)=0, \quad j=0,1, \cdots, n-1 \\
P_{n} \nu(a)=P_{n} \mu(a)-P_{n} \mu_{1}(a)=A+D_{n+1}^{*} F(a)=0, \\
P_{n+1} \nu(x)=P_{n+1} \mu(x)-F_{1}(x) \geqq 0 .
\end{array}\right.
$$

These relations imply by $(0.5)$ that $\nu \in\left[C\left(u_{0}, \cdots, u_{n}\right) \cap C\left(u_{0}, \cdots, u_{n+1}\right)\right]^{*}$ so that the decomposition has been accomplished and the theorem is proved.

It is quite surprising, knowing Theorems 2 and 3 , that the corresponding statement concerning four consecutive convexity cones is false. This is demonstrated by the following example which shows that

$$
\begin{aligned}
& {\left[C(1) \cap C(1, x) \cap C\left(1, x, x^{2}\right) \cap C\left(1, x, x^{2}, x^{3}\right)\right]^{*}} \\
& \quad \neq C^{*}(1)+C^{*}(1, x)+C^{*}\left(1, x, x^{2}\right)+C^{*}\left(1, x, x^{2}, x^{3}\right) .
\end{aligned}
$$


EXAMPLE. Let the measure $\mu$ be represented by the function $f(t)=-\int_{a}^{t} d \mu$, where

$$
f(t)=\left\{\begin{array}{cl}
400 t-40, & 0.1 \leqq t<0.2 \\
80-200 t, & 0.4 \leqq t<0.5 \\
200 t-120, & 0.5 \leqq t<0.6 \\
40-50 t, & 0.6 \leqq t<0.8 \\
0 \quad, & \text { elsewhere } .
\end{array}\right.
$$

It is immediately seen that

$$
\int_{0}^{1} f(t) d t=1, \quad \int_{0}^{1} t f(t) d t=0
$$

and a simple computation yields

$$
\int_{0}^{1} t^{2} f(t) d t>0
$$

Consider the function $Q(x)$ defined by

$$
Q(x)=\int_{x}^{1}(t-x)^{2} f(t) d t, \quad 0 \leqq x \leqq 1 .
$$

Differentiation yields

$$
Q^{\prime}(x)=-2 \int_{x}^{1}(t-x) f(t) d t, \quad Q^{\prime \prime}(x)=2 \int_{x}^{1} f(t) d t .
$$

The second derivative $Q^{\prime \prime}(x)$ has in $(0,1)$ precisely two zeros, as can be directly checked from the graph of $f(t)$. Since $Q^{\prime}(x)$ vanishes for 0 and 1 , it may have at most one zero inside $(0,1)$. Finally, since $Q(0)>0$ by $(2.22)$, and $Q(1)=0$ while $Q(x)$ is positive near 1 , we may deduce that $Q(x) \geqq 0$ for all $0 \leqq x \leqq 1$. Using the fact that $\left\{(t-x)_{+}^{2}\right\}$ are the extreme rays for $C\left(1, x, x^{2}\right)$, we deduce by $(0.3)$ that $f(t) \in\left[C^{+} \cap C(1) \cap C(1, x) \cap C\left(1, x, x^{2}\right)\right]^{*}$. Since $f(1)=0$, this implies that $\mu \in\left[C(1) \cap C(1, x) \cap C\left(1, x, x^{2}\right) \cap C\left(1, x, x^{2}, x^{3}\right)\right]^{*}$ (cf. [6]).

Suppose now that there exists a decomposition $\mu=\mu_{1}+\mu_{2}$ with $\mu_{1} \in C^{*}(1), \mu_{2} \in\left[C(1, x) \cap C\left(1, x, x^{2}\right) \cap C\left(1, x, x^{2}, x^{3}\right)\right]^{*}$, then there exists a corresponding decomposition $f=f_{1}+f_{2}$ where $f_{i}(t)=-\int_{a}^{t} d \mu_{i}$, $i=1,2 ; f_{1} \in\left(C^{+}\right)^{*}, f_{2} \in\left[C(1) \cap C(1, x) \cap C\left(1, x, x^{2}\right)\right]^{*}$.

By (0.3), these functions have to satisfy

$$
\begin{cases}f_{1}(x) \geqq 0 & 0 \leqq x \leqq 1 \\ \int_{0}^{1} f_{2}(t) d t=0, & \int_{0}^{1} t f_{2}(t) d t \geqq 0 .\end{cases}
$$


In this case, however, the equalities

$$
0=\int_{0}^{1} t f(t) d t=\int_{0}^{1} t f_{1}(t) d t+\int_{0}^{1} t f_{2}(t) d t
$$

imply that

$$
\int_{0}^{1} t f_{1}(t) d t=0
$$

Since $f_{1}(t)$ is nonnegative and continuous from the right, it follows that $f_{1}(t) \equiv 0$. On the other hand, we know that, by $(2.21)$ and $(2.23)$,

$$
1=\int_{0}^{1} f(t) d t=\int_{0}^{1} f_{1}(t) d t
$$

Thus, we have arrived at a contradiction, which shows that the assumption that there exists a decomposition is untenable.

3. Sums of dual cones in $M_{0}$. The existence of a decomposition of $\mu \in M_{0} \cap\left[C\left(u_{0}, \cdots, u_{n-1}\right) \cap C\left(u_{0}, \cdots, u_{n}\right)\right]^{*}$ into a sum of a measure $\mu_{1} \in M_{0} \cap C^{*}\left(u_{0}, \cdots, u_{n-1}\right)$ and a measure $\mu_{2} \in M_{0} \cap C^{*}\left(u_{0}, \cdots, u_{n}\right)$ follows from Theorem 2. For these cones, however, we have the stronger result:

THEOREM 4. Let $\mu$ be a measure of $\left[C\left(u_{0}, \cdots, u_{n-1}\right) \cap C\left(u_{0}, \cdots, u_{n}\right)\right]^{*}$ such that inf $\{$ support $(\mu)\}=s>a$. Then there exists a decomposition $\mu=\mu_{1}+\mu_{2}, \mu_{1} \in C^{*}\left(u_{0}, \cdots, u_{n-1}\right), \mu_{2} \in C^{*}\left(u_{0}, \cdots, u_{n}\right)$ where $\mu_{1}$ is either 0 or supported in any $n+1$ prescribed distinct points in $(a, s)$.

Proof. We may assume that

$$
\int_{a}^{b} u_{n}(t) d \mu(t)=A>0
$$

since otherwise $\mu \in C^{*}\left(u_{0}, \cdots, u_{n}\right)$ and the decomposition is accomplished by choosing $\mu_{1}=0$.

Let $\left\{x_{i}\right\}_{1}^{n+1}$ be an arbitrarily prescribed set of points such that $a<x_{1}<\cdots<x_{n+1}<s$. Consider the system of linear equations

$$
\left\{\begin{array}{l}
\sum_{j=1}^{n+1} a_{j} u_{i}\left(x_{j}\right)=0, \quad i=0,1, \cdots, n-1 \\
\sum_{j=1}^{n+1} a_{j} u_{n}\left(x_{j}\right)=A .
\end{array}\right.
$$

Since $\left(u_{0}, \cdots, u_{n}\right)$ is a Tchebycheff system, the corresponding determinant does not vanish, and the system of equations has a unique solution $\left(a_{1}, \cdots, a_{n+1}\right)$. 
Define $\mu_{1}=\sum_{j=1}^{n+1} a_{j} \delta_{x_{j}}$, where $\delta_{x_{j}}$ is the Dirac measure with unit mass at $x_{j}$. By virtue of (3.1), we have

$$
\begin{gathered}
\int_{a}^{b} u_{i}(t) d \mu_{1}(t)=0, \quad i=0,1, \cdots, n-1 . \\
\int_{a}^{b} u_{n}(t) d \mu_{1}(t)=A>0 .
\end{gathered}
$$

From [6], Theorem B, we deduce that $\mu_{1}$ has at least $n$ sign changes. Since it is supported in at most $n+1$ points, it follows that it has exactly $n$ sign changes. The same theorem allows us to conclude that either $\mu_{1}$ or $-\mu_{1}$ belong to $C^{*}\left(u_{0}, \cdots, u_{n-1}\right)$. Since $u_{n} \in C\left(u_{0}, \cdots, u_{n-1}\right)$, relation (3.3) implies that $\mu_{1} \in C^{*}\left(u_{0}, \cdots, u_{n-1}\right)$.

It remains to be shown that $\mu_{2}=\mu-\mu_{1}$ belongs to $C^{*}\left(u_{0}, \cdots, u_{n}\right)$. It is easy to deduce that

$$
\int_{a}^{b} u_{i}(t) d \mu_{2}(t)=0, \quad i=0,1, \cdots, n .
$$

Thus, by (0.3), we have to show only that

$$
\int_{a}^{b} \phi_{n}(t ; x) d \mu_{2}(t) \geqq 0, \quad a<x<b .
$$

Consider first the case $x>x_{n+1}$. We have

$$
\int_{a}^{b} \phi_{n}(t ; x) d \mu_{1}(t)=\int_{x}^{b} \phi_{n}(t ; x) d \mu_{1}(t)=0
$$

so that

$$
\int_{a}^{b} \phi_{n}(t ; x) d \mu_{2}(t)=\int_{a}^{b} \phi_{n}(t ; x) d \mu(t) \geqq 0 .
$$

The inequality following by applying (0.3) to the measure $\mu$. Suppose next that $x \leqq x_{n+1}$. Since $x_{n+1}<s$, we have

$$
\begin{aligned}
\int_{a}^{b} \phi_{n}(t ; x) d \mu(t) & =\int_{x}^{b} \phi_{n}(t ; x) d \mu(t) \\
& =\int_{x}^{b}\left[u_{n}(t)+\sum_{i=0}^{n-1} c_{i}(x) u_{i}(t)\right] d \mu(t) \\
& =\int_{x}^{b} u_{n}(t) d \mu(t)+\sum_{i=0}^{n-1} c_{i}(x) \int_{x}^{b} u_{i}(t) d \mu(t) \\
& =\int_{a}^{b} u_{n}(t) d \mu(t)+\sum_{i=0}^{n-1} c_{i}(x) \int_{a}^{b} u_{i}(t) d \mu(t)=A .
\end{aligned}
$$

The last equality following from (3.2)-(3.3).

Thus, for $x \leqq x_{n+1}$, (3.5) is equivalent to 


$$
\int_{a}^{b} \phi_{n}(t ; x) d \mu_{1}(t) \leqq A
$$

Since

$$
\begin{aligned}
& \int_{a}^{b}\left[\phi_{n}(t ; x)+(-1)^{n} \tilde{\phi}_{n}(t ; x)\right] d \mu_{1}(t) \\
& \quad=\int_{a}^{b}\left[u_{n}(t)+\sum_{i=0}^{n-1} c_{i}(x) u_{i}(t)\right] d \mu_{1}(t)=A .
\end{aligned}
$$

We further deduce that (3.6) is equivalent to

$$
\int_{a}^{b}(-1)^{n} \widetilde{\phi}_{n}(t ; x) d \mu_{1}(t) \geqq 0
$$

which follows from the fact that $(-1)^{n} \tilde{\phi}_{n}(t ; x) \in C\left(u_{0}, \cdots, u_{n-1}\right)$ and $\mu_{1} \in C^{*}\left(u_{0}, \cdots, u_{n-1}\right)$. This completes the proof of Theorem 4 .

4. Application. In this section we derive a simple RiemannStieltjes integration scheme which converges monotonely for all convex functions. We start by restating a theorem of Fejer (see [4]):

Given 2 interlaced partitions, $\left(s_{i}\right)_{i=0}^{m},\left(t_{i}\right)_{i=0}^{m+1}$, of the interval [0,1]: $0=t_{0}=s_{0}<t_{1}<s_{1}<t_{2}<\cdots<s_{i}<t_{i+1}<s_{i+1}<\cdots<t_{m}<s_{m}=$ $t_{m+1}=1$. Form the Riemann sums:

$$
\begin{gathered}
S_{m}(g)=\sum_{i=1}^{m}\left(s_{i}-s_{i-1}\right) g\left(s_{i}\right) \\
S_{m+1}(g)=\sum_{i=1}^{m+1}\left(t_{i}-t_{i-1}\right) g\left(t_{i}\right) .
\end{gathered}
$$

Then necessary and sufficient conditions for

$$
S_{m+1}(g) \leqq S_{m}(g)
$$

to hold for every increasing and convex function $g$ on $[0,1]$, are:

$$
\sum_{i=0}^{k} q_{i}\left(p_{i}-p_{i+1}\right) \geqq 0 \quad(i=0, \cdots, m-1)
$$

where $p_{i}=s_{m-i}-t_{m-i}, q_{i}=t_{m-i}-s_{m-i-1}$. (To derive this formulation from the theorem as stated in [5], substitute: $g(x)=f(1-x)$, $\left.m=n+1, s_{i}=1-x_{m-i}, t_{i}=1-y_{m+1-i}\right)$. Furthermore, it was shown in [5] that no Riemann scheme of this type converges monotonely for all monotone function or for all convex functions. In order to obtain a scheme converging monotonely for all convex functions we shall modify Fejer's scheme to a Riemann-Stieltjes scheme.

The inequality (4.1) means that, provided (4.2) is satisfied, the measure $\mu$ defined by $\mu=\sum_{i=1}^{m}\left(s_{i}-s_{i-1}\right) \delta_{s_{i}}-\sum_{i=1}^{m+1}\left(t_{i}-t_{i-1}\right) \delta_{t_{i}}$ belongs to $\left[C(1) \cap C(1, x]^{*}\right.$. We apply to $\mu$ the decomposition of Theorem 4: Given 2 points $u, v$ satisfying: $0<u<v<t_{1}$, we construct the meas- 
ure $\mu_{1}=A\left(\delta_{v}-\delta_{u}\right) /(v-u)$, where $A=\int_{0}^{1} t d \mu(t)=\sum_{i=0}^{m-1} q_{i}\left(p_{i}-p_{i+1}\right) \quad$ (cf. [4.2]). Then, we have $\mu-\mu_{1} \in C^{*}(1, x)$. This means that, for every convex function $g$, we have:

$$
S_{n}(g)-\frac{A}{v-u} g(v) \geqq S_{n+1}(g)-\frac{A}{v-u} g(u) .
$$

Suppose now we have a sequence of consecutively interlaced partitions $\Delta^{m}=\left(\tau_{i}^{m}\right)_{i=0}^{m}(m=1,2, \cdots)$, such that:

$$
\begin{aligned}
& 0=\tau_{0}^{m+1}=\tau_{0}^{m}<\tau_{1}^{m+1}<\tau_{1}^{m}<\cdots<\tau_{i}^{m+1}<\tau_{i}^{m}<\tau_{i+1}^{m+1}<\cdots<\tau_{m}^{m} \\
& =\tau_{m+1}^{m+1}=1 \quad(m=1,2, \cdots)
\end{aligned}
$$

and $\sum_{i=0}^{k} q_{i}^{m}\left(p_{i}^{m}-p_{i+1}^{m}\right) \geqq 0(k=0, \cdots, m-1 ; m=1,2, \cdots)$ where $p_{i}^{m}=$ $\tau_{m-i}^{m}-\tau_{m-i}^{m+1}, q_{i}^{m}=\tau_{m-i}^{m+1}-\tau_{m-i-1}^{m}$, and suppose also we have a sequence $\left(v_{i}\right)$ satisfying: $0<v_{m+1}<v_{m}<\tau_{1}^{m+1}(m=0,1, \cdots)$ and

$$
A_{m} /\left(v_{m}-v_{m+1}\right)=\gamma(\text { constant })(m=1,2, \cdots) \text { where } A_{m}=\sum_{i=0}^{m-1} q_{i}^{m}\left(p_{i}^{m}-p_{i+1}^{m}\right) \text {. }
$$

Define:

$$
\sum_{m}(g)=\sum_{i=1}^{m}\left(\tau_{i}^{m}-\tau_{i-1}^{m}\right) g\left(\tau_{i}\right)-\gamma g\left(v_{m}\right) .
$$

Then the sequence $\sum_{m}(g)$ decreases monotonely for every convex function $g$, the limit being $\int_{0}^{1} g(t) d t-\gamma g(0)$, provided the partitions $\Delta^{m}$ tend to zero (in maximal diameter). A simple useful scheme satisfying our requirements is: $\tau_{i}^{m}=i / m(i=0, \cdots, m ; m=1,2, \cdots), v_{m}=1 / 2 m$. In this scheme $A_{m}=1 / 2 m(m+1)$ and $\gamma=1$, hence for every function $g$ convex on $[0,1]$, the sums $\sum_{i=1}^{m} g(i / m) / m-g(1 / 2 m)$ decrease monotonely to $\int_{0}^{1} g(t) d t-g(0)$.

\section{REFERENCES}

1. R. E. Edwards, Functional Analysis, Holt, New York 1965.

2. S. Karlin and A. Novikoff, Generalized convex inequalities, Pacific J. Math. 13 (1963), 1251-1279.

3. S. Karlin and W. Studden, Tchebycheff systems with applications in Analysis and Statistics, Interscience, New York, 1966.

4. G. Szegö and P. Turan, On the monotone convergence of certain Riemann sums, Publ. Math. Debrecen 8 (1961), 326-335.

5. Z. Ziegler, Generalized convexity cones, Pacific J. Math. 17 (1966), 561-580.

6. - On the characterization of measures of the cone dual to a generalized convexity cone, Pacific J. Math. 24 (1968), 603-626.

Received August 1, 1967. 


\section{PACIFIC JOURNAL OF MATHEMATICS}

\section{EDITORS}

\section{H. ROYDEN}

Stanford University

Stanford, California

\section{J. Dugundu}

Department of Mathematics University of Southern California Los Angeles, California 90007

RICHARD ARENS

University of California Los Angeles, California 90024

ASSOCIATE EDITORS
E. F. Beckenbach
B. H. NEUMANN
F. WoLF
K. YosidA

\section{SUPPORTING INSTITUTIONS}

\author{
UNIVERSITY OF BRITISH COLUMBIA \\ CALIFORNIA INSTITUTE OF TECHNOLOGY \\ UNIVERSITY OF CALIFORNIA \\ MONTANA STATE UNIVERSITY \\ UNIVERSITY OF NEVADA \\ NEW MEXICO STATE UNIVERSITY \\ OREGON STATE UNIVERSITY \\ UNIVERSITY OF OREGON \\ OSAKA UNIVERSITY \\ UNIVERSITY OF SOUTHERN CALIFORNIA
}

STANFORD UNIVERSITY

UNIVERSITY OF TOKYO

UNIVERSITY OF UTAH

WASHINGTON STATE UNIVERSITY

UNIVERSITY OF WASHINGTON

AMERICAN MATHEMATICAL SOCIETY
CHEVRON RESEARCH CORPORATION
TRW SYSTEMS

AMERICAN MATHEMATICAL SOCIETY

TRW SYSTEMS

NAVAL WEAPONS CENTER

Mathematical papers intended for publication in the Pacific Journal of Mathematics should be in typed form or offset-reproduced, double spaced with large margins. Underline Greek letters in red, German in green, and script in blue. The first paragraph or two must be capable of being used separately as a synopsis of the entire paper. It should not contain references to the bibliography. Manuscripts, in duplicate if possible, may be sent to any one of the four editors. All other communications to the editors should be addressed to the managing editor, Richard Arens, University of California, Los Angeles, California 90024.

Each author of each article receives 50 reprints free of charge; additional copies may be obtained at cost in multiples of 50 .

The Pacific Journal of Mathematics is published monthly. Effective with Volume 16 the price per volume (3 numbers) is $\$ 8.00$; single issues, $\$ 3.00$. Special price for current issues to individual faculty members of supporting institutions and to individual members of the American Mathematical Society: $\$ 4.00$ per volume; single issues $\$ 1.50$. Back numbers are available.

Subscriptions, orders for back numbers, and changes of address should be sent to Pacific Journal of Mathematics, 103 Highland Boulevard, Berkeley 8, California.

Printed at Kokusai Bunken Insatsusha (International Academic Printing Co., Ltd.), 7-17, Fujimi 2-chome, Chiyoda-ku, Tokyo, Japan.

PUBLISHED BY PACIFIC JOURNAL OF MATHEMATICS, A NON-PROFIT CORPORATION

The Supporting Institutions listed above contribute to the cost of publication of this Journal, but they are not owners of publishers and have no responsibility for its content or policies. 


\section{Pacific Journal of Mathematics \\ Vol. 27, No. 3}

March, 1968

Charles A. Akemann, Invariant subspaces of $C(G) \ldots \ldots \ldots \ldots \ldots \ldots . \ldots 41$

Dan Amir and Zvi Ziegler, Generalized convexity cones and their duals ... . 425

Raymond Balbes, On ( $J, M, \mathrm{~m})$-extensions of order sums of distributive

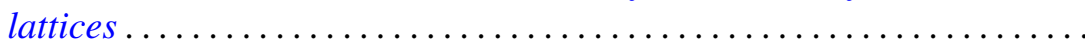

Jan-Erik Björk, Extensions of the maximal ideal space of a function algebra ........................................ 453

Frank Castagna, Sums of automorphisms of a primary abelian group ...... 463

Theodore Seio Chihara, On determinate Hamburger moment problems ..... .

Zeev Ditzian, Convolution transforms whose inversion function has complex

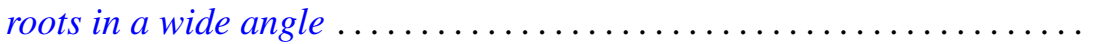

Myron Goldstein, On a paper of Rao .

Velmer B. Headley and Charles Andrew Swanson, Oscillation criteria for

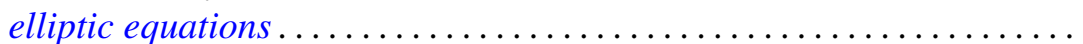

John Willard Heidel, Qualitative behavior of solutions of a third order nonlinear differential equation............................

Alan Carleton Hindmarsh, Pick's conditions and analyticity.............

Bruce Ansgar Jensen and Donald Wright Miller, Commutative semigroups

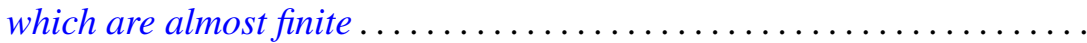

Lynn Clifford Kurtz and Don Harrell Tucker, An extended form of the

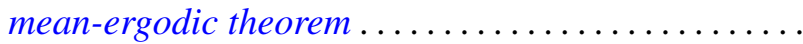

S. P. Lloyd, Feller boundary induced by a transition operator ...

Henry B. Mann, Josephine Mitchell and Lowell Schoenfeld, A new proof of the maximum principle for doubly-harmonic functions ...

Robert Einsohn Mosher, The product formula for the third obstruction ..

Sam Bernard Nadler, Jr., Sequences of contractions and fixed points ...

Eric Albert Nordgren, Invariant subspaces of a direct sum of weighted shifts...

Fred Richman, Thin abelian p-groups ...

Jordan Tobias Rosenbaum, Simultaneous interpolation in $\mathrm{H}_{2}$. II ...

Charles Thomas Scarborough, Minimal Urysohn spaces .

Malcolm Jay Sherman, Disjoint invariant subspaces..... .

Joel John Westman, Harmonic analysis on groupoids....

621

William Jennings Wickless, Quasi-isomorphism and TFM

Minoru Hasegawa, Correction to "On the convergence of resolvents of operators" 